\title{
Comparison of sample preparation methods for the identification of Staphylococcus Aureus by MALDI-FOF MS
}

\author{
${ }^{1}$ Brigitta Horváth $-{ }^{1}$ Ferenc Peles $-{ }^{2}$ László Bereczki $-{ }^{2}$ András Széll $-{ }^{3}$ Rita Sipos $-{ }^{3}$ Ágnes Erôs - \\ ${ }^{4}$ Csaba Lovász $-{ }^{4}$ Adrienn Micsinai \\ ${ }^{1}$ Institute of Food Science, Faculty of Agricultural and Food Sciences and Environmental Management, University of Debrecen, \\ Böszörményi str. 138, 4032 Debrecen, Hungary \\ ${ }^{2}$ MikroMikoMed Ltd. H-1036 Budapest Lajos str. 74-76. Hungary \\ ${ }^{3}$ BIOMI Ltd. H-2100 Gödöllő Szent-Györgyi Albert str. 4 Hungary \\ ${ }^{4}$ WESSLING Hungary Ltd. H-1045 Budapest Anonymus str. 6. Hungary \\ horvath.brigitta920108@gmail.com
}

\section{SUMMARY}

Coagulase-positive staphylococci include 3 species, Staphylococcus aureus, S. hyicus and S. intermedius. Of these three species, S. aureus is the most well-known human pathogen. S. aureus is part of the human and animal normal microbiota, however, it is capable of producing several staphylococcal enterotoxins (SEs) that cause intoxication symptoms of varying intensity in humans after consuming contaminated food. Selective media which are used for the determination of coagulase-positive staphylococci from foods are not able to identify isolates at a species. With the MALDI-TOF MS technique, we can identify S. aureus cheaper and faster than by using molecular methods. This paper describes the results of the study of the presence of coagulase-positive staphylococci and $\mathrm{S}$. aureus in many food products, and the application of three sample preparation methods: direct sample preparation, formic acid suspension and ethanol extraction.

Keywords: Coagulase-positive staphylococci, MALDI-TOF MS, sample preparation, foodborne pathogen, S. aureus

\section{INTRODUCTION}

Staphylococcus is the main genus of the Staphylococcaceae family in the order of Bacillales, Bacilli class and Firmicutes phylum. Staphylococci are non-spore forming, non-motile, Gram-positive cocci, 0.5 to 1 micrometer in diameter, catalase and coagulase positive.

Coagulase-positive staphylococci include 3 species, S. aureus, S. hyicus and S. intermedius (ANSES, 2011; EURL CPS, 2014). The most virulent pathogen species of the genus Staphylococcus is S. aureus (Foster, 1996) (Jain and Daum, 1999).

Staphylococcus aureus is facultatively anaerobic, and can be found on the skin and mucous membranes of $20-30 \%$ of people and warm-blood animals. These microbes have also been isolated in the natural environment, hospital environment and foodstuff (Manukumar and Umesha, 2017). Staphylococcus aureus forms golden-yellow colonies on blood agar (Taylor and Unakal, 2017) and produces several toxins, including staphylococcal enterotoxins responsible for staphylococcal food-poisoning outbreaks (Ercoli et al., 2017).

Many S. aureus biotypes have been isolated from various hosts (human, poultry, cattle and sheep/goat) that show a close adaptation of the microorganism to the host cell (Hennekinne et al., 2012). The most common cause of subclinical mastitis in cattle are coagulase-positive staphylococci, but other animals (pigs, poultry, horses) can also be infected. Therefore, these microorganisms may often be present in foods (Rajic-Savic et al., 2015).

Staphylococcal food poisoning is one of the most common food-borne illnesses (Jain and Daum, 1999) (Kadariya et al., 2014). Enterotoxigenic strains of coagulase-positive staphylococci produce enterotoxins, mainly Staphylococcus aureus and very rarely other Staphylococcus species, such as Staphylococcus intermedius (Loir et al., 2003). Staphylococcal food poisoning symptoms develop rapidly (2-8 hours), including nausea, vomiting, abdominal cramps, with or without diarrhea. The disease usually does not require treatment and typically resolves after 24 to 48 hours of recovery (Kadariya et al., 2014). Occasionally, the infection may prove to be serious enough to require hospital treatment, especially when infants or elderly are affected (Argudín et al., 2010).

In order to ensure the microbiological safety of food, both EU level and national regulation exists. Regulation (EC) No. 2073/2005 sets criteria - among others - for coagulase-positive staphylococci in several food product categories of animal origins as process hygiene parameters to be investigated by the set of EN ISO 6888 norms. National Hungarian regulation (Ministry of Health regulation No. 4/1998) also sets microbiological limits. Of these, Staphylococcus aureus is prescribed as a technological limit criterion with regard to several foodstuffs with constituents of animal origin.

Selective media which are used for the determination of coagulase-positive staphylococci from foods are not able to identify isolates at a species. Thus, it is important to develop such new methods for identification that allow reduction of time-to-result for the food and feed industry. The aim was to look into the possibility of applying MALDI-TOF MS technique for the identification of coagulase-positive staphylococci.

The matrix-assisted laser desorption ionizationtime of flight mass spectrometry (MALDI-TOF MS) technique provide information on the protein and macromolecule profiles of the sample. Obtained mass 
spectra serve as base for the routine identification of the microbes, compared to a validated database (Singhal et al., 2015) to uncover markers or marker sets which can reliably identify Staphylococcus species (Pavlovic et al., 2013). Furthermore, this technique provides information about the microorganism variety of sources: e.g. isolates from clinical, livestock, food, feed or environmental sources (Sandrine et al., 2012).

\section{MATERIALS AND METHODS}

\section{Bacterial strains, isolates and culture conditions}

In the present study, 20 coagulase-positive staphylococci isolates were collected from different foodstuffs (Table 1). Based on the MSZ EN ISO 68881:2008 standard, the isolates were isolated by culturing on selective and non-selective growth media. On BairdParker selective medium (Biokar, Fr), staphylococci formed typical colonies and all strains showed positive coagulase reaction. For the coagulase test (Microgen Bio Product, UK), colonies were grown at $37{ }^{\circ} \mathrm{C}$ for $24 \pm 1 \mathrm{~h}$ on Columbia Blood agar (Neogen, UK). The strains and their sources are indicated in Table 1.

The sources of the different Staphylococcus spp. strains isolated from food matrices exhibiting typical features on Baird-Parker medium and testing positive in coagulase latex test

\begin{tabular}{|c|c|c|c|}
\hline \multicolumn{2}{|l|}{ Category of food } & ID number & Type of food sample \\
\hline \multirow{3}{*}{ Milk products } & & SA-1 & Cheese \\
\hline & & SA-10 & Cheese \\
\hline & & SA-18 & Milk \\
\hline \multirow{2}{*}{ Dried pasta } & & SA-2 & Dried pasta \\
\hline & & SA-7 & Dried pasta \\
\hline \multirow{15}{*}{ Meat } & & SA-12 & Chicken wings \\
\hline & & SA-13 & Duck liver \\
\hline & Poultry & SA-16 & Chicken breast \\
\hline & & SA-17 & Goose liver \\
\hline & & SA-20 & Duck meat \\
\hline & & SA-4 & Bacon \\
\hline & & SA-5 & Pork sausage \\
\hline & & SA-6 & Pork chops \\
\hline & Pork & SA-9 & Pork shoulder \\
\hline & & SA-14 & Pork sausage \\
\hline & & SA-15 & Pork greaves \\
\hline & & SA-19 & Pork chops \\
\hline & & SA-3 & Beef \\
\hline & Beef & SA-8 & Beef \\
\hline & & SA-11 & Beef \\
\hline
\end{tabular}

\section{Sample preparation}

For identification with the MALDI-TOF MS technique, the isolates were grown on Columbia Blood agar. Three different types of sample preparation for MALDI-TOF MS analysis were used. The direct sample preparation was based on the standard Bruker Daltonic Inc. protocol. In this case, samples were taken from colonies with sterile sampling loops, then transferred directly onto the target plate and $1-1 \mu 170$ $\mathrm{v} / \mathrm{v} \%$ formic acid was added to them. After drying, $1 \mu \mathrm{l}$ $\alpha$-HCCA $(10 \mathrm{mg} / \mathrm{ml})$ matrix solution was added to the samples and the spots were crystallized by air drying.

In the second sample preparation protocol, formic acid suspending was used: a single colony was picked up with an inoculation loop and it was suspended in 40 $\mu \mathrm{l}$ of formic acid in an Eppendorf tube for 30 seconds. As a next step, $40 \mu \mathrm{l}$ of acetonitrile was added to the suspension and mixed thoroughly. Finally, $1 \mu \mathrm{l}$ of the suspension was transferred onto the target plate and when dried, it was overlaid with $1 \mu$ l $\alpha$-HCCA (10 $\mathrm{mg} / \mathrm{ml}$ ) matrix solution and left to dry again.

In the third sample preparation protocol, ethanol extraction was used: a single colony was suspended in $300 \mu \mathrm{l}$ of distilled water in an Eppendorf tube. Next, $900 \mu \mathrm{l}$ absolute ethanol was added to the suspension, vortexed for 30 seconds and centrifuged at 14,500 rpm $(18188 \mathrm{x} \mathrm{g})$ for 3 minutes. After the supernatant was removed, the pellet was resuspended in $40 \mu \mathrm{l}$ of 70 $\mathrm{v} / \mathrm{v} \%$ formic acid. Following this, $40 \mu \mathrm{l}$ acetonitrile was added to the suspension, mixed and centrifuged thoroughly. Finally, $1 \mu \mathrm{l}$ of the suspension was transferred onto the target plate, and after the sample dried, $1 \mu \mathrm{l}$ of $\alpha$-HCCA $(10 \mathrm{mg} / \mathrm{ml})$ was added.

\section{MALDI-TOF MS measurement parameters}

Mass spectra were obtained by the application of Bruker Microflex LT MALDI-TOF mass spectrometer operating in positive linear mode, in the molecular 
mass range of $2.0-25 \mathrm{kDa}$. We used the MALDI BioTyper 3.1 software to identify Staphylococcus spp. More than 200 shots gave adequate spectra with appropriate signal-to-noise ratio. Before the measurement, the calibration was carried out with Bruker Bacterial Test Standard. For the aim of analysis, 640 shots were performed mass spectra of Staphylococcus spp. Following the measurement, the mass data files were transferred to flexAnalysis 3.4 software (Bruker Daltonics). All spectra were processed by baseline correction, Gaussian smoothing, and peak finding. The obtained mass spectra were analysed individually for characteristic peaks.

The 20 isolates were analysed in parallel, the obtained mass spectra were compared to MALDI Bruker's Biotyper-specific database of mass spectra. The obtained results are reported as numeric score values based on similarity with the reference spectra. Scores below 1.699 reported as non-reliable genus identification, scores of 1.700-1.999 were classified as probable genus identification, scores of 2.000-2.299 were secure genus identification and scores of 2.3003.000 designated as highly probable species identification.

\section{RESULTS AND DISCUSSION}

\section{Identification Staphylococcus spp.}

By using the direct sample preparation method, it was possible to obtain identification scores higher than 1.700 in the case of all $(100 \%)$ isolates by comparison with the Bruker MALDI-Biotyper database. Seven Staphylococcus spp. isolates gave score values $\geq 2.300$, therefore, the species Staphylococcus aureus was safely identified. Eleven isolates had score values between 2.000 and 2.300, i.e., the isolates' genus level identification was secure. Two strains had a probable genus level identification with a score in the 1.7001.999 range.

The summary of the identification results of the direct sample preparation protocol including the best and second best matching organism names and scores are shown in Table 2. According to Manukumar et al. (2017), similar results were obtained when foodstuffs were investigated: 34 out of 36 coagulase positive Staphylococcus isolates were identified with a $>2.000$ score value, and two isolates had score values between 1.700-1999 when direct sample preparation was used.

Score values of identification for the direct sample preparation method

\begin{tabular}{|c|c|c|c|c|}
\hline Analyte name & Organism (best match) & Score value & Organism (second best match) & Score value \\
\hline SA-1 & Staphylococcus aureus & 2.185 & Staphylococcus aureus & 2.156 \\
\hline SA-2 & Staphylococcus aureus & 2.214 & Staphylococcus aureus & 2.115 \\
\hline SA-3 & Staphylococcus aureus & 2.219 & Staphylococcus aureus & 2.047 \\
\hline SA-4 & Staphylococcus aureus & 2.278 & Staphylococcus aureus & 2.124 \\
\hline SA-5 & Staphylococcus aureus & 2.158 & Staphylococcus aureus & 2.023 \\
\hline SA-6 & Staphylococcus aureus & 2.391 & Staphylococcus aureus & 2.264 \\
\hline SA-7 & Staphylococcus aureus & 2.372 & Staphylococcus aureus & 2.195 \\
\hline SA-8 & Staphylococcus aureus & 2.136 & Staphylococcus aureus & 2.102 \\
\hline SA-9 & Staphylococcus aureus & 2.271 & Staphylococcus aureus & 2.224 \\
\hline SA-10 & Staphylococcus aureus & 2.233 & Staphylococcus aureus & 2.172 \\
\hline SA-11 & Staphylococcus aureus & 2.416 & Staphylococcus aureus & 2.370 \\
\hline SA-12 & Staphylococcus aureus & 2.441 & Staphylococcus aureus & 2.326 \\
\hline SA-13 & Staphylococcus aureus & 2.374 & Staphylococcus aureus & 2.211 \\
\hline SA-14 & Staphylococcus aureus & 2.413 & Staphylococcus aureus & 2.409 \\
\hline SA-15 & Staphylococcus aureus & 1.838 & Staphylococcus aureus & 1.795 \\
\hline SA-16 & Staphylococcus aureus & 2.158 & Staphylococcus aureus & 2.141 \\
\hline SA-17 & Staphylococcus aureus & 2.213 & Staphylococcus aureus & 2.182 \\
\hline SA-18 & Staphylococcus aureus & 1.813 & Staphylococcus aureus & 1.804 \\
\hline SA-19 & Staphylococcus aureus & 2.252 & Staphylococcus aureus & 2.167 \\
\hline SA-20 & Staphylococcus aureus & 2.356 & Staphylococcus aureus & 2.249 \\
\hline
\end{tabular}

The results of the formic acid suspension sample preparation did not differ from the direct suspension method. Seven coagulase-positive staphylococci isolates gave score values $\geq 2.300$, eleven isolates had score values between 2.000 and 2.300 , and two strains had scores in the 1.700-1.999 range (Table 3.). The SA15 and SA-18 isolates had the lowest score values for both of the two sample preparation methods.
The results of the ethanol extraction sample preparation method did not differ fundamentally from the direct and formic acid suspension methods. In this case, eight coagulase-positive staphylococci isolates had score values $\geq 2.300$, nine isolates had score values between 2.000 and 2.300, and three strain gave scores in the 1.700-1.999 range. The best and second best matching organism names and scores are shown in Table 4. 
Score values of identification for the formic acid suspending sample preparation method

\begin{tabular}{|c|c|c|c|c|}
\hline Analyte name & Organism (best match) & Score value & Organism (second best match) & Score value \\
\hline SA-1 & Staphylococcus aureus & 2.238 & Staphylococcus aureus & 2.163 \\
\hline SA-2 & Staphylococcus aureus & 2.252 & Staphylococcus aureus & 2.118 \\
\hline SA-3 & Staphylococcus aureus & 2.217 & Staphylococcus aureus & 2.209 \\
\hline SA-4 & Staphylococcus aureus & 2.246 & Staphylococcus aureus & 2.231 \\
\hline SA-5 & Staphylococcus aureus & 2.188 & Staphylococcus aureus & 2.162 \\
\hline SA-6 & Staphylococcus aureus & 2.429 & Staphylococcus aureus & 2.344 \\
\hline SA-7 & Staphylococcus aureus & 2.365 & Staphylococcus aureus & 2.307 \\
\hline SA-8 & Staphylococcus aureus & 2.216 & Staphylococcus aureus & 2.081 \\
\hline SA-9 & Staphylococcus aureus & 2.273 & Staphylococcus aureus & 2.268 \\
\hline SA-10 & Staphylococcus aureus & 2.172 & Staphylococcus aureus & 2.132 \\
\hline SA-11 & Staphylococcus aureus & 2.405 & Staphylococcus aureus & 2.386 \\
\hline SA-12 & Staphylococcus aureus & 2.312 & Staphylococcus aureus & 2.309 \\
\hline SA-13 & Staphylococcus aureus & 2.333 & Staphylococcus aureus & 2.215 \\
\hline SA-14 & Staphylococcus aureus & 2.461 & Staphylococcus aureus & 2.447 \\
\hline SA-15 & Staphylococcus aureus & 1.887 & Staphylococcus aureus & 1.829 \\
\hline SA-16 & Staphylococcus aureus & 2.215 & Staphylococcus aureus & 2.081 \\
\hline SA-17 & Staphylococcus aureus & 2.211 & Staphylococcus aureus & 2.094 \\
\hline SA-18 & Staphylococcus aureus & 1.866 & Staphylococcus aureus & 1.837 \\
\hline SA-19 & Staphylococcus aureus & 2.252 & Staphylococcus aureus & 2.164 \\
\hline SA-20 & Staphylococcus aureus & 2.373 & Staphylococcus aureus & 2.271 \\
\hline
\end{tabular}

Score values of identification for the ethanol extraction sample preparation method

\begin{tabular}{|c|c|c|c|c|}
\hline Analyte name & Organism (best match) & Score value & Organism (second best match) & Score value \\
\hline SA-1 & Staphylococcus aureus & 2.281 & Staphylococcus aureus & 2.194 \\
\hline SA-2 & Staphylococcus aureus & 2.301 & Staphylococcus aureus & 2.297 \\
\hline SA-3 & Staphylococcus aureus & 2.124 & Staphylococcus aureus & 2.119 \\
\hline SA-4 & Staphylococcus aureus & 1.797 & Staphylococcus aureus & 1.786 \\
\hline SA-5 & Staphylococcus aureus & 2.195 & Staphylococcus aureus & 2.073 \\
\hline SA-6 & Staphylococcus aureus & 2.396 & Staphylococcus aureus & 2.308 \\
\hline SA-7 & Staphylococcus aureus & 2.331 & Staphylococcus aureus & 2.310 \\
\hline SA-8 & Staphylococcus aureus & 2.127 & Staphylococcus aureus & 2.065 \\
\hline SA-9 & Staphylococcus aureus & 2.162 & Staphylococcus aureus & 2.115 \\
\hline SA-10 & Staphylococcus aureus & 2.164 & Staphylococcus aureus & 2.034 \\
\hline SA-11 & Staphylococcus aureus & 2.376 & Staphylococcus aureus & 2.084 \\
\hline SA-12 & Staphylococcus aureus & 2.336 & Staphylococcus aureus & 2.311 \\
\hline SA-13 & Staphylococcus aureus & 2.412 & Staphylococcus aureus & 2.319 \\
\hline SA-14 & Staphylococcus aureus & 2.462 & Staphylococcus aureus & 2.343 \\
\hline SA-15 & Staphylococcus aureus & 1.793 & Staphylococcus aureus & 1.728 \\
\hline SA-16 & Staphylococcus aureus & 2.253 & Staphylococcus aureus & 2.192 \\
\hline SA-17 & Staphylococcus aureus & 2.187 & Staphylococcus aureus & 2.037 \\
\hline SA-18 & Staphylococcus aureus & 1.913 & Staphylococcus aureus & 1.879 \\
\hline SA-19 & Staphylococcus aureus & 2.210 & Staphylococcus aureus & 2.201 \\
\hline SA-20 & Staphylococcus aureus & 2.382 & Staphylococcus aureus & 2.259 \\
\hline
\end{tabular}

Table 5 summarizes the results of the different sample preparation methods. The direct sample preparation and the formic acid suspension yielded basically the same identification results. Seven strains gave score values $\geq 2.300$, eleven isolates had score values between 2.000 and 2.300 , and two isolates had the lowest score values for each of the two sample preparation methods. The results of the ethanol method differed slightly from the results of the other two methods. In that case eight isolates had score values $\geq$ 2.300 , nine strains gave score values between $2.000-$ 2.300, and three isolates gave scores in the 1.700-1.999 range. 
Summary table of result of identification

\begin{tabular}{lccc}
\cline { 2 - 4 } & & Sample preparation & \\
\hline Score value & Direct sample preparation & Formic acid suspending & Ethanol extraction \\
\hline $2.300 \leq$ & 7 & 7 & 8 \\
$2.000-2.300$ & 11 & 11 & 9 \\
$1.700-1.999$ & 2 & 2 & 3 \\
\hline
\end{tabular}

Table 6 summarizes the best score value of mean and deviation of different sample preparation. The best score value of mean and deviation were defined using Microsoft Excel 2007. The largest deviation was obtained in SA-4 sample (0.269), the smallest standard deviation was found at the SA-20 sample (0.013). The largest mean of score values was determined in the SA14 sample (2.445), the lowest mean of score value was determined in the SA-15 sample.

Summary table of mean and deviation of best score value

\begin{tabular}{|c|c|c|c|c|c|}
\hline $\begin{array}{l}\text { Analyte } \\
\text { name }\end{array}$ & $\begin{array}{l}\text { Best score of direct } \\
\text { sample preparation }\end{array}$ & $\begin{array}{c}\text { Best score of formic acid } \\
\text { suspension }\end{array}$ & $\begin{array}{c}\text { Best score of ethanol } \\
\text { extraction }\end{array}$ & $\begin{array}{c}\text { Mean of score } \\
\text { value }\end{array}$ & $\begin{array}{c}\text { Standard deviation } \\
\text { of score value }\end{array}$ \\
\hline SA-1 & 2.185 & 2.238 & 2.281 & 2.235 & 0.048 \\
\hline SA-2 & 2.214 & 2.252 & 2.301 & 2.256 & 0.044 \\
\hline SA-3 & 2.219 & 2.217 & 2.124 & 2.187 & 0.054 \\
\hline SA-4 & 2.278 & 2.246 & 1.797 & 2.107 & 0.269 \\
\hline SA-5 & 2.158 & 2.188 & 2.195 & 2.180 & 0.020 \\
\hline SA-6 & 2.391 & 2.429 & 2.396 & 2.405 & 0.021 \\
\hline SA-7 & 2.372 & 2.365 & 2.331 & 2.356 & 0.022 \\
\hline SA-8 & 2.136 & 2.216 & 2.127 & 2.160 & 0.049 \\
\hline SA-9 & 2.271 & 2.273 & 2.162 & 2.235 & 0.064 \\
\hline SA-10 & 2.233 & 2.172 & 2.164 & 2.190 & 0.038 \\
\hline SA-11 & 2.416 & 2.405 & 2.376 & 2.399 & 0.021 \\
\hline SA-12 & 2.441 & 2.312 & 2.336 & 2.363 & 0.069 \\
\hline SA-13 & 2.374 & 2.333 & 2.412 & 2.373 & 0.040 \\
\hline SA-14 & 2.413 & 2.461 & 2.462 & 2.445 & 0.028 \\
\hline SA-15 & 1.838 & 1.887 & 1.793 & 1.839 & 0.047 \\
\hline SA-16 & 2.158 & 2.215 & 2.253 & 2.209 & 0.048 \\
\hline SA-17 & 2.213 & 2.211 & 2.187 & 2.204 & 0.014 \\
\hline SA-18 & 1.813 & 1.866 & 1.913 & 1.864 & 0.050 \\
\hline SA-19 & 2.252 & 2.252 & 2.210 & 2.238 & 0.024 \\
\hline SA-20 & 2.356 & 2.373 & 2.382 & 2.370 & 0.013 \\
\hline
\end{tabular}

The difference in the summarised results of the ethanol extraction method to the other two methods with regard to the number of strains showed two strains switching identification levels. Strain SA-2 was securely identified at a species level (score value 2.301) with the ethanol extraction method contrary to the other two method which offered only secure genus level identification. However, the standard deviation of score values for the SA-2 strain between the three methods is smaller than the average standard deviation of the 20 strains (0.049). Consequently, this change in identification might not be significant. The other strain that had its identification levels changed when using the ethanol extraction method is SA-4. This strain's score was 2.278 and 2.246 with the direct sample preparation and the formic acid suspension testing, respectively, whereas the ethanol extraction gave only a 1.797 score. The reason for this difference is not well understood, maybe a protein rich fraction's behaviour is behind the phenomenon.

\section{CONCLUSIONS}

The MALDI-TOF MS technique can be used to assess the taxonomic position of coagulase-positive staphylococci from several sources, including foodstuffs. Based on result of direct sample preparation and the formic acid suspension sample preparation 19 out of 20 coagulase-positive Stapylococcus isolates were securely identified at genus level and seven isolates were identified at species level with a high probability. During ethanol extraction sample preparation, eight isolates were identified at species level with a high probability, but three strains had a score in the 1.700-1.999 range, therefore, in these cases, the identification of genus failed. Based on a first 
and second best score value, all isolates were evaluated as Staphylococcus aureus.

Two sample preparation methods (direct and formic acid techniques) yielded the same identification evaluation results. The result of the third sample preparation method (ethanol suspension technique) is slightly different from the other two processes, therefore, this procedure needs to be investigated for the causes of the discrepancy, since the quality of the obtained spectrum of isolates can affect the score value of identification.

The MALDI-TOF MS technique is a simple, quick and exact tool for a more reliable and even faster identification and confirmation of the taxonomic position of coagulase-positive staphylococci and, more specifically, of Staphylococcus aureus. This method can be implemented into routine diagnostic microbiology due to its high throughput and fast timeto-result capabilities. With the use of the MALDI-TOF technique, streaking of typical or suspect colonies on nutrient agar and the classical biochemical confirmations could be replaced, thus the analysis time of Staphylococcus spp. could be reduced by at least 24 hours.

\section{ACKNOWLEDGEMENTS}

The study was supported by the EFOP-3.6.3VEKOP-16-2017-00008 project. The project is cofinanced by the European Union and the European Social Fund.

\section{REFERENCES}

Argudín, M. Á.-Mendoza, M. C.-Rodicio, M. R. (2010): Food Poisoning and Staphylococcus aureus Enterotoxins. Toxins. 7. 1751-1773.

Ercoli, L.-Gallina, S.-Nia, Y.-Auvray, F.-Primavilla, S.-Guidi, F.Pierucci, B.-Graziotti, C.-Decastelli, L.-Scuota, S. (2017): Investigation of a Staphylococcal Food Poisoning Outbreak from a Chantilly Cream Dessert, in Umbria (Italy). Foodborne Pathogenes and Disease 14. 407-413.

European Union Reference Laboratory for Coagulase Positive Staphylococci- EURL CPS (2014): About Coagulase Positive Staphylococci. (2018.11.12.) https://sites.anses.fr/en/minisite/staphylococci/about-coagulasepositive-staphylococci

Foster, T. (1996): Staphylococcus. Chapter 12. Medical Microbiology.

French Agency for Food, Environmental and Occupational Health \& Safety- ANSES (2011): Staphylococcus aureus and staphylococcal enterotoxins. (2018. 11. 13.) https://www.anses.fr/fr/system/files/MIC2011sa0117FiEN_0.p df

Hennekinne, J. A.-De Buyser, M. L.-Dragacci, S. (2012): Staphylococcus aureus and its food poisoning toxins: characterization and outbreak investigation. FEMS Microbiology Reviews. 36. 815-836.

Jain, A.-Daum, S. R. (1999): Staphylococcal Infections in Children: Part. Pediatrics in Review. 20. 183-191.

Kadariya, J.-Smith, T. C.-Thapaliya, D. (2014): Staphylococcus aureus and Staphylococcal Food-Borne Disease: An Ongoing Challenge in Public Health. Biomed Research International. 2014. 1-9.
Loir, L. Y.-Baron, F.-Gautier, M. (2003): Staphylococcus aureus and food poisoning. Genetics and Molecular Research. 2. 63-76.

Manukumar, H. M.-Umesha, S. (2017): MALDI-TOF-MS based identification and molecular characterization of food associated methicillin-resistant Staphylococcus aureus. Scientific Reports. 7. 1-16.

Pavlovic, M.-Huber, I.-Konrad, R.-Busch, U. (2013): Application of MALDI-TOF MS for the Identification of Food Borne Bacteria. The Open Microbiology Journal. 7. 135-141.

Rajic-Savic, N.-Katic, V.-Velebit, B.-Colovic, S. (2015): Characteristics of enterotoxigenic coagulase positive staphylococci isolated from bovine milk in cases of subclinical mastitis. Procedia Food Science. 5. 250-253.

Sandrin, T. R.-Goldstein, J. E.-Schumaker, S. (2012): MALDI TOF MS profiling of bacteria at the strain level: A review. Mass Spectrometry Reviews. 32. 188-217.

Singhal, N.-Kumar, M.-Kanaujia, P. K.-Virdi, J. S. (2015): MALDITOF mass spectrometry: an emerging technology for microbial identification and diagnosis. Frontiers in microbiology. 791. +116.

Taylor, T. A.-Unakal, C. G. (2017): Staphylococcus Aureus. StatPearls Publishing. (2018. $11 . \quad 12$. https://www.ncbi.nlm.nih.gov/books/NBK441868/ 